\title{
Clinical Findings of Pelvic Inflammatory Diseases among women in Reproductive Age
}

\author{
Ismat Ara Laizuㄹ, Laila Anjuman Banu², Fowzia Abul Fayez, \\ ${ }^{1}$ Senior Consultant, Department of Gynaecology \& Obstetrics, Mugda Medical College \& Hospital, Dhaka, Bangladesh. \\ ${ }^{2}$ Associate professor (Gynae and obs), Mugda Medical College, Dhaka, Bangladesh; ${ }^{3}$ Junior Consultant (Gynae), \\ Muradnagar Upazila Health Complex, Muradnagar, Cumilla, Banalgdesh.
}

[Received on: 22 November 2020; Accepted on: 12 December 2020; Published: 1 January 2021]

\begin{abstract}
Background: Pelvic Inflammatory disease is a very common gynaecological condition among the women in reproductive age group. Objectives: The purpose of the present study was to find out the clinical findings of Pelvic inflammatory diseases among the women during reproductive age group. Methodology: This cross-sectional study was carried out in the Department of Obstetrics and Gynaecology at Mymensingh Medical College Hospital, Mymensingh, Bangladesh from January 2008 to June 2009 for a period of one (01) and a half year. Women who were presented with pelvic inflammatory disease (PID) attended at the OPD of gynecology Department at Mymensingh Medical College Hospital, Mymensingh, Bangladesh were selected as study population. Detailed clinical history of each patient was taken and thorough physical examination was performed. Result: A total number of 300 cases were recruited. The mean age with SD was $30.3 \pm 9.57$ years. Majority of the patients had tenderness in the lower abdomen which was 96(64.0\%) cases. However, 152(50.7\%) patients had vaginal discharge and 34(11.3\%) patients had uterovaginal proplapse. However, cervical tear was present in 96(32.0\%) cases and chronic cervicitis was present in 138(46.0\%) cases. Conclusion: In conclusion most common clinical feature is lower abdominal tenderness followed by vaginal discharge. [Journal of National Institute of Neurosciences Bangladesh, January 2021;7(1): 52-55]
\end{abstract}

Keywords: : Clinical Findings; Pelvic Inflammatory Diseases; Reproductive Age

Correspondence: Dr. Ismat Ara Laizu, Senior Consultant, Department of Gynaecology \& Obstetrics, Mugda Medical College \& Hospital, Dhaka, Bangladesh. E-mail: laizuismat@gmail.com; Cell no.: +8801785872929

Conflict of interest: There is no financial conflict of interest relevant to this paper to disclose.

Funding agency: This research project was not funded by any group or any institution.

Contribution to authors: Fayz FA contributed from the protocol preparation, data collection up to report writing. Manuscript writing was performed by Fayz FA have revised the manuscript.

How to cite this article: Laizu IA, Banu LA, Fayz FA. Clinical Findings of Pelvic Inflammatory Diseases among women in Reproductive Age. J Natl Inst Neurosci Bangladesh, 2021;7(1): 52-55

Copyright: (C2021. Laizu et al. Published by Journal of National Institute of Neurosciences Bangladesh. This article is published under the Creative Commons CC BY-NC License (https://creativecommons.org/licenses/by-nc/4.0/). This license permits use, distribution and reproduction in any medium, provided the original work is properly cited, and is not used for commercial purposes.

\section{Introduction}

Pelvic Inflammatory Disease (PID) is an ascending infection in which pathogenic microorganisms ascend from the lower genital tract to the upper genital tract ${ }^{1}$. Endometritis is an early manifestation of PID and most but not all women with PID have plasma cell endometritis ${ }^{2}$. Therefore, salpingitis develops, which can lead to pyosalpinx or tubo-ovarian abscess formation. Perihepatitis is associated with PID in $10-20 \%$ of cases ${ }^{3}$. It is a poly-microbial disease. Variety of organisms cause pelvic sepsis with superadded secondary infection and that's why the primary pathogen remains unidentified. Although gonorrhoea has long been considered the major cause of pelvic inflammatory disease, however, the studies have found a rising proportion of non-gonococcus pelvic inflammatory disease ${ }^{4}$ By improved culture technique, workers have isolated Chlamydia trachomatis, anaerobic bacteria, enterobacteriae and Mycoplasma hominis as bacterial pathogens of pelvic infection, but the relative importance of these as primary or secondary pathogen is uncertain ${ }^{5}$. Anaerobic organisms are important as a secondary 
invader than a primary cause of pelvic inflammatory disease, but the possibility that they may initiate the infection is not excluded ${ }^{6}$. The ascent of micro-organisms from the lower genital tract to the upper genital tract is the cause of pelvic inflammatory disease. One theory suggests that pelvic inflammatory disease usually starts as a cervical infection and is followed by production of metabolic waste products that alter the cervico-vaginal micro-environment ${ }^{7}$. This result in overgrowth of facultative anaerobic flora, which then ascend, with or without the original cervical pathogen, into the endometrium, fallopian tube and peritoneal cavity ${ }^{8}$. The purpose of the present study was to find out the clinical findings of pelvic inflammatory diseases among the women during reproductive age group.

\section{Methodology}

This study was designed as descriptive cross-sectional study. This study was carried out in the Department of Obstetrics and Gynaecology at Mymensingh Medical College Hospital, Mymensingh, Bangladesh from January 2008 to June 2009 for a period of one (01) and a half year. Women at any age who were suffering from pelvic inflammatory disease (PID) attended at the OPD of gynecology Department at Mymensingh Medical College Hospital, Mymensingh, Bangladesh were selected as study population. Clinically the patients were diagnosed with the presence of at least three of the symptoms like chronic pelvic pain or backache, deep dyspareunia, congestive dysmenorrhea, menstrual irregularities as well as the signs like lower abdominal tenderness, cervical motion tenderness and adnexal tenderness with or without thickening of fornices or mass. Detailed clinical history of each patient was taken and thorough physical examination was performed. Epidemiological aspects and clinical presentation have been mainly highlighted in this study. Relevant data from each patient were recorded in a questionnaire. Data were analyzed by SPSS version 21.0 software package. All data were recorded systematically in a preformed data collection sheet. The quantitative data were expressed as frequency and percentage and the quantitative data were expressed as mean with standard deviation.

\section{Results}

A total number of 300 cases were recruited after fulfilling the inclusion and exclusion criteria. Among 300 cases of pelvic inflammatory disease, majority (54.0\%) belonged to the age group of 26 to 35 years followed by 18 to 25 years and more than 35 years age group which were $100(33.3 \%)$ cases and $38(12.7 \%)$ cases respectively. The mean age with SD was $30.3 \pm 9.57$ with the range of minimum 18 years and maximum 49 years (Table 1 ).

Table 1: Age Distribution of the Patients $(n=300)$

\begin{tabular}{lcc}
\hline Age Group & Frequency & Percent \\
\hline 18 to 25 Years & 100 & 33.3 \\
26 to 35 Years & 162 & 54.0 \\
More than 35 Years & 38 & 12.7 \\
Total & $\mathbf{3 0 0}$ & $\mathbf{1 0 0 . 0}$ \\
Mean \pm SD (Range) & $30.3 \pm 9.57$ (18 to 49) \\
\hline
\end{tabular}

Majority of the patients had tenderness in the lower abdomen which was 96(64.0\%) cases. However. Lower abdominal mass was found in only $6(4.0 \%)$ cases of study population. The rest of $48(32.0 \%)$ cases had no abnormalities during per abdominal examination (Table 2).

Table 2: Per abdominal Examination Findings $(\mathrm{n}=300)$

\begin{tabular}{lcc}
\hline Findings in Lower Abdomen & Frequency & Percent \\
\hline Tenderness & 96 & 64.0 \\
Mass & 6 & 4.0 \\
No Abnormalities & 48 & 32.0 \\
Total & $\mathbf{1 5 0}$ & $\mathbf{1 0 0 . 0}$ \\
\hline
\end{tabular}

In this study 152(50.7\%) patients had vaginal discharge and $34(11.3 \%)$ patients had uterovaginal prolapse either

TTable 3: Findings of Per Vaginal Examination among Study Population $(\mathrm{n}=300)$

\begin{tabular}{lcc}
\hline Variables & Frequency & Percent \\
\hline Vaginal Discharge & 152 & 50.7 \\
Uterovaginal Prolapse & 34 & 11.3 \\
Cervical tear & 96 & 32.0 \\
Chronic cervicitis & 138 & 46.0 \\
Cervical motion tenderness & 212 & 70.7 \\
Position of Uterus & & \\
- Anteverted $\quad 190$ & 63.3 \\
- Retroverted $\quad 110$ & 36.7 \\
Mobility of Uterus & & \\
- Mobile & 140 & 46.7 \\
- Restricted & 96 & 32.0 \\
- Fixed & 66 & 22.0 \\
Tenderness of fornices & 290 & 96.7 \\
Thickening of fornices & 206 & 68.7 \\
Tuboovarian Mass & 16 & 5.3 \\
\hline
\end{tabular}




\section{Discussion}

Clinical features of pelvic inflammatory disease may vary in developed countries ${ }^{8}$. It is more common among women with multiple sexual partners and women who report a history of STD compared with those with no STD history. The most important presenting feature is chronic pelvic pain of varying magnitude. The pain is dull in nature and continuously present over the both lower abdominal quadrants9. The pain aggravates prior to and during menstruation due to congestion. Pain is also increased with movement, coitus and micturition. There may be a sensation of pelvic pressure radiating down one or both legs; however, it is deep seated and occur due to the pelvic cellulites, especially when there is involvement of uterosacral ligament ${ }^{10}$.

The detailed and methodical study of 300 cases in this series shows highest $(54.3 \%)$ incidence of this disease being in the age group of 26 to 35 years. Khan et $\mathrm{al}^{11}$ have also showed that women with PID are usually under the age of 25 years. Simms et $\mathrm{al}^{12}$ showed that $87.0 \%$ patients belong to the age group 20 to 35 years. There is similarity between this last study which was conducted in India, with the present study. Laila ${ }^{13}$ also showed that $55.21 \%$ of her patients were in the age group of 26 to 35 years. PID occurs more in younger age group in western countries where the disease is mainly STD related, but in developing countries, it is mostly non-STD related and occurs in later age group ${ }^{11}$. Chronic pelvic inflammatory disease is never seen in prepubertal women and very rarely after the menopause. The most frequent age of involvement is between 15 and 25 years. This peak age reflect the sexual activities of this group of women. Patients are mostly multiparous. Most of them are having no antenatal checkup and have a home delivery which is conducted by relatives or dais?. There may be pelvic infection following child birth; however, many of the patients have a previous history of spontaneous or induced abortion or MR by unskilled person ${ }^{14}$.

Younger age is marked by biological characteristics conductive to the development of PID, such as a lower prevalence of protective chlamydial antibody, larger zone of cervical ecotypy and greater permeability of cervical mucosa ${ }^{15}$. The correlation between early coital indulgence and promiscuous sexual relationship might explain the very high saplingitis incidence in sexually active teenage girls. However, STD is less important for development of PID in the somewhat older women. In this age group of patients and also in women who have had two or more episodes of PID, anaerobic bacteria is thought to be the aetiological agent. The reason behind this may be the post PID fallopian tubes are more vulnerable to infections by anaerobes ${ }^{13}$. Anatomic changes induced by pregnancy and delivery contribute to an easier access to the vagina for bowel flora; furthermore, this may lead to an increased occurrence of a type of non-veneral PID in women of comparatively higher age ${ }^{16}$.

Major symptoms for which the patients of this series reported to the obstetric and gynaecological outpatient department, Dhaka Medical College Hospital, in order of frequency, are lower abdominal pain (96.0\%), backache $(78.6 \%)$, dyspareunia $(76.6 \%)$, congestive dysmenorrhoea (64\%), vaginal discharge $(60.0 \%)$ and sterility $(6.7 \%)$, they also complained of menstrual abnormalities in the form of menorrhagia (20\%), polymenorrhoea $(6.7 \%)$, polymenorrhagia $(2.7 \%)$, dysmenorrhoea (72.7\%). Laila ${ }^{13}$ has shown that $95.31 \%$ of her patients had chronic lower abdominal pain. Sultana ${ }^{16}$ showed that all her patients had lower abdominal pain. In another study ${ }^{17}$ it has been reported that the most frequent clinical symptoms are some form of menstrual disturbances, $48.0 \%$ women complained of lower abdominal pain, $31.1 \%$ women experienced vaginal discharge.

The vaginal discharge is almost a constant manifestation. The discharge is mucoid or mucopurulent in nature and it is related to the congestion and associated cervical pathology ${ }^{18}$. Congestive dysmenorrhoea occurs due to pelvic congestion associated with premenstrual vascular engorgement. Backache is due to chronic cellulitis in uterine ligaments and becomes exaggerated during menstruation. There may be menorrhagia due to congestion and at times epimenorrhagia due to ovarian involvement. Patient may complain of infertility which is generally secondary in type. The factors causing infertility are cornual block, loss of cilia, loss of peristalsis due to thickening of tubal wall, closure of the abdominal ostium and distortion of the tube due to peritubal adhesion ${ }^{19}$. Patient may complain of deterioration of general health due to presence of a septic foci.

The past or presence of intrauterine contraceptive device is corroborative. An increasing number of women with PID will not have classical features. Chlamydia as well as gonococci will be found in asymptomatic women. There may be low grade temperature with tachycardia. There may be tenderness in the lower abdomen both iliac fossa, hypogastrium; however, irregular tender pelvic mass may be felt. In others, no palpable abnormality is detected except 
tenderness ${ }^{13}$.

In this study a good number of cases had cervical tear. This indicates the spread of infection to the upper genital tract from the lower genital tract. A large number of patients showed features of chronic cervicitis. Majority of the patients showed evidence of pelvic cellulitis on bimanual examination. Adnexal mass was palpated in 6 patients. Following an acute pelvic infection, there was healing by fibrosis. This results in kinking of tubes which get adherent to the ovaries, uterus, intestine, omentum and pelvic peritoneum. This leads to the formation of tuboovarian mass. During per vaginal examination, there may be variable degrees of perineal and cervical tear. The cervix may show features of chronic cervicitis. In bimanual examination, the uterus may be bulky with restricted mobility or may be fixed retroversion. The cervix may descend down to some extent. The tear and descend helps in ascending infection ${ }^{17}$. Cervix may be congested and patulous. Pain will be present on moving the cervix during pelvic examination. Fornices reveal tenderness and thickening or even a mass on one or both sides. In long-standing cases, there may be tuboovarian mass extending to involve the pouch of Doglus. Rectal examination corroborates the findings of vaginal examination and should not be omitted especially when the vaginal examination is painful ${ }^{13}$.

\section{Conclusion}

In conclusion most of the patients are presented with tenderness in the lower abdomen. However. Lower abdominal mass is also found in majority of the study population. In addition vaginal discharge is also reported in a significant number of women. A large number of patients are presented with uterovaginal prolapse either in the form of cystocele, rectocele or uterine prolapse. However, cervical tear is also reported. Furthermore, the features of chronic cervicitis is also reported. During bimanual examination, cervical motion tenderness is also found. Fornices are found tender and thickened in a significant number of women presented with pelvic inflammatory diseases.

\section{References}

1. Soper DE. Pelvic inflammatory disease. Obstetrics \& Gynecology. 2010;116(2):419-28.

2. Tewiri PV, Neelam MO. A study of lukol in leucorrhoea, pelvic inflammatory diseases and dysfunctional uterine bleeding. Ancient Science of life. 2001;21(2):139.
3. Kreisel K, Torrone E, Bernstein K, Hong J, Gorwitz R. Prevalence of pelvic inflammatory disease in sexually experienced women of reproductive age-United States, 2013-2014. MMWR. Morbidity and mortality weekly report. 2017;66(3):80.

4. Ness RB, Soper DE, Holley RL, Peipert J, Randall H, Sweet RL, Sondheimer SJ, Hendrix SL, Amortegui A, Trucco G, Songer T. Effectiveness of inpatient and outpatient treatment strategies for women with pelvic inflammatory disease: results from the Pelvic Inflammatory Disease Evaluation and Clinical Health (PEACH) Randomized Trial. American journal of obstetrics and gynecology. 2002;186(5):929-37.

5. Simms I, Stephenson JM. Pelvic inflammatory disease epidemiology: what do we know and what do we need to know? Sexually transmitted infections. 2000;76(2):80-7.

6. McCormack WM. Pelvic inflammatory disease. New England Journal of Medicine. 1994;330(2):115-9.

7. Taylor BD, Darville T, Haggerty CL. Does bacterial vaginosis cause pelvic inflammatory disease?. Sexually transmitted diseases. 2013;40(2):117-22.

8. Simms I, Stephenson JM, Mallinson H, Peeling RW, Thomas K, Gokhale R, Rogers PA, Hay P, Oakeshott P, Hopwood J, Birley H. Risk factors associated with pelvic inflammatory disease. Sexually transmitted infections. 2006;82(6):452-7.

9. Haggerty CL, Ness RB. Epidemiology, pathogenesis and treatment of pelvic inflammatory disease. Expert review of anti-infective therapy. 2006;4(2):235-47.

10. Mitchell C, Prabhu M. Pelvic inflammatory disease: current concepts in pathogenesis, diagnosis and treatment. Infectious Disease Clinics. 2013;27(4):793-809.

11. Khan N, Rahman F, Jahan N, Khatun R, Saha N. Clinical Profiles of Pelvic Inflammatory Disease Patients: Experience of 150 Cases at a Tertiary Care Hospital in Dhaka City. Journal of National Institute of Neurosciences Bangladesh. 2018;4(2):129-32 12. Simms I, Stephenson JM, Mallinson H, Peeling RW, Thomas K, Gokhale R, Rogers PA, Hay P, Oakeshott P, Hopwood J, Birley H. Risk factors associated with pelvic inflammatory disease. Sexually transmitted infections. 2006;82(6):452-7

13. Laila R. Study of 192 cases of chronic PID in GOPD of Dhaka Medical College Hospital (dissertation), Dhaka: Bangladesh College of Physicians and Surgeons, 1997

14. French CE, Hughes G, Nicholson A, Yung M, Ross JD, Williams T, Soldan K. Estimation of the rate of pelvic inflammatory disease diagnoses: trends in England, 2000-2008. Sexually transmitted diseases. 2011;38(3):158-62

15. Sutton MY, Sternberg M, Zaidi A, Louis ME, Markowitz LE. Trends in pelvic inflammatory disease hospital discharges and ambulatory visits, United States, 1985-2001. Sexually transmitted diseases. 2005;32(12):778-84

16. Sultana S, Aetiological aspect of chronic PID: a case report of 349 cases in GOPD (dissertation), Dhaka: Bangladesh College of Physicians and Surgeons, 1996

17. Gray-Swain MR, Peipert JF. Pelvic inflammatory disease in adolescents. Current Opinion in Obstetrics and Gynecology. 2006;18(5):503-10

18. Peterson HB. Galaid EI, Cater W. Pelvic inflammatory disease, Med clin N Am 1990; 74: 1603-5.

19. Tai FW, Chang CY, Chiang JH, Lin WC, Wan L. Association of pelvic inflammatory disease with risk of endometriosis: a nationwide cohort study involving 141,460 individuals. Journal of clinical medicine. 2018;7(11):379 